\title{
M-QAM Based Single-Carrier Baseband Communication System Research
}

\author{
Aboagye Isaac Adjaye ${ }^{1}$, Okoampa Boadu Edwin ${ }^{2}$ \\ ${ }^{1}$ (Communication and Information Engineering, University of Electronic Science and Technology of China, \\ Ghana) \\ ${ }^{2}$ (Micro Electronics Engineering, University of Electronic Science and Technology of China, Ghana)
}

\begin{abstract}
All transmitted signals are to some extent degraded by the environment through which they propagate. Signal degradation can take many forms, but generally it falls into three types: noise, distortion, and attenuation (reduction in power). In order to solve or reduce these errors generated during signal transmission, an M-QAM single-carrier baseband communication system was designed and simulations were made to determine the BER under different SNR. This method of modulation has the advantage of reducing or eliminating intermodulation interference caused by a continuous carrier near the modulation sidebands. Also it will enable data transmission at twice the rate of standard pulse amplitude modulation (PAM) without any degradation in the bit error rate (BER), thereby doubling the effective bandwidth. The numerical results obtained from the Matlab simulink were compared to the theoretical results obtained from the Matlab tool named 'bertool' when $Q A M$ orders for $E_{b} / N_{0}$ were set at $12.0 \mathrm{~dB}$, the new simulated BER's for the different QAM's were obtained. The BER values obtained from both simulation systems were found to be similar. Keywords: MQAM, System parameters, Communication System, AWGN, Matlab Simulink.
\end{abstract}

\section{Introduction}

Communications systems are designed to transmit information generated by a source to some destination ${ }^{[1]}$. All transmitted signals are to some extent degraded by the environment through which they propagate. Signal degradation can take many forms, but generally it falls into three types: noise, distortion, and attenuation (reduction in power). Errors are also generated in communication and transmission of information due to the inaccurate estimation of carrier phase and frequency performed at the receiver. These errors can affect the quality if received signals.

Ronghong Mo and Yong Huat Chew published a paper on Capacity of Rate Adaptive M-QAM Systems in the Presence of Channel Estimation Errors under BER Constraint. They addressed the problem of deriving the SNR threshold intervals, given the statistical characteristics of channel fading and estimation error. The capacity of adaptive MQAM systems was then investigated. They also showed that with estimation errors, some higher order constellation cannot be used since it cannot meet the BER requirement and hence results in significant capacity loss.

Since information transmitted are sometimes or mostly corrupted by noise and other interferences, quadrature amplitude modulation (QAM) as a means of modulation ensures effective transmission of information. In this way there is a balance between obtaining the higher data rates and maintaining an acceptable bit error rate for any radio communications system ${ }^{[2]}$. The quadrature amplitude modulation (QAM) has become common place in bandwidth efficient digital communication systems. It conveys two analog message signals, or two digital bit streams, by changing (modulating) the amplitudes of two carrier waves, using the amplitude-shift keying (ASK) digital modulation scheme or amplitude modulation (AM) analog modulation scheme ${ }^{[3]}$.

The design and implementation of M-QAM in a communication system as this paper portrays will ensure an effective way of transmission and retrieval of information in communication systems. It will also contribute to the wealth of knowledge already in existence and will empower researchers to carry out further studies in all relevant areas of knowledge as far as M-QAM is concerned.

\subsection{Signal Constellation}

A signal constellation is the physical diagram used to describe all the possible symbols used by a signaling system to transmit data and is an aid to designing better communications systems. It helps in designing a transmission system that is less prone to errors and can possibly recover from transmission problems without relying on higher level protocols. The measured constellation diagrams were used to recognize the type of interference and distortion in a signal. 
Error probability for MQAM is:

\section{Qam Ber Calculation}

$$
P_{e}=1-P_{c}=2 P_{\sqrt{M}}-P_{\sqrt{M}}^{2} \leq 2 P_{\sqrt{M}}=\frac{4(\sqrt{M}-1)}{\sqrt{M}} Q\left(\sqrt{\frac{3}{M-1} \times \frac{E_{a v}}{N_{0}}}\right)
$$

$P_{\sqrt{M}}$ is small ,so $P_{e} \approx 2 P_{\sqrt{M}}$. When using Gray coding, each symbol error causes only a little bit of error. This is the bit error rate performance of the system to the top. Thus:

$$
P_{b} \approx \frac{P_{e}}{\log _{2} M} \approx \frac{2 P_{\sqrt{M}}}{\log _{2} M}=\frac{4(\sqrt{M}-1)}{\sqrt{M} * \log _{2} M} Q\left(\sqrt{\frac{3}{M-1} \times \frac{E_{a v}}{N_{0}}}\right) .
$$

\section{System Analysis And Design}

The design system was built using the simulink tool integrated in the Matlab environment. The various components for the system design were obtained from the communication system toolbox via the Matlab library and sampled together. The system was modeled to represent dynamic system that reacted to the changing channel conditions. Each subsystem serves as a unit test harness for the other. Scope was used to monitor the simulation results while the simulation runs.

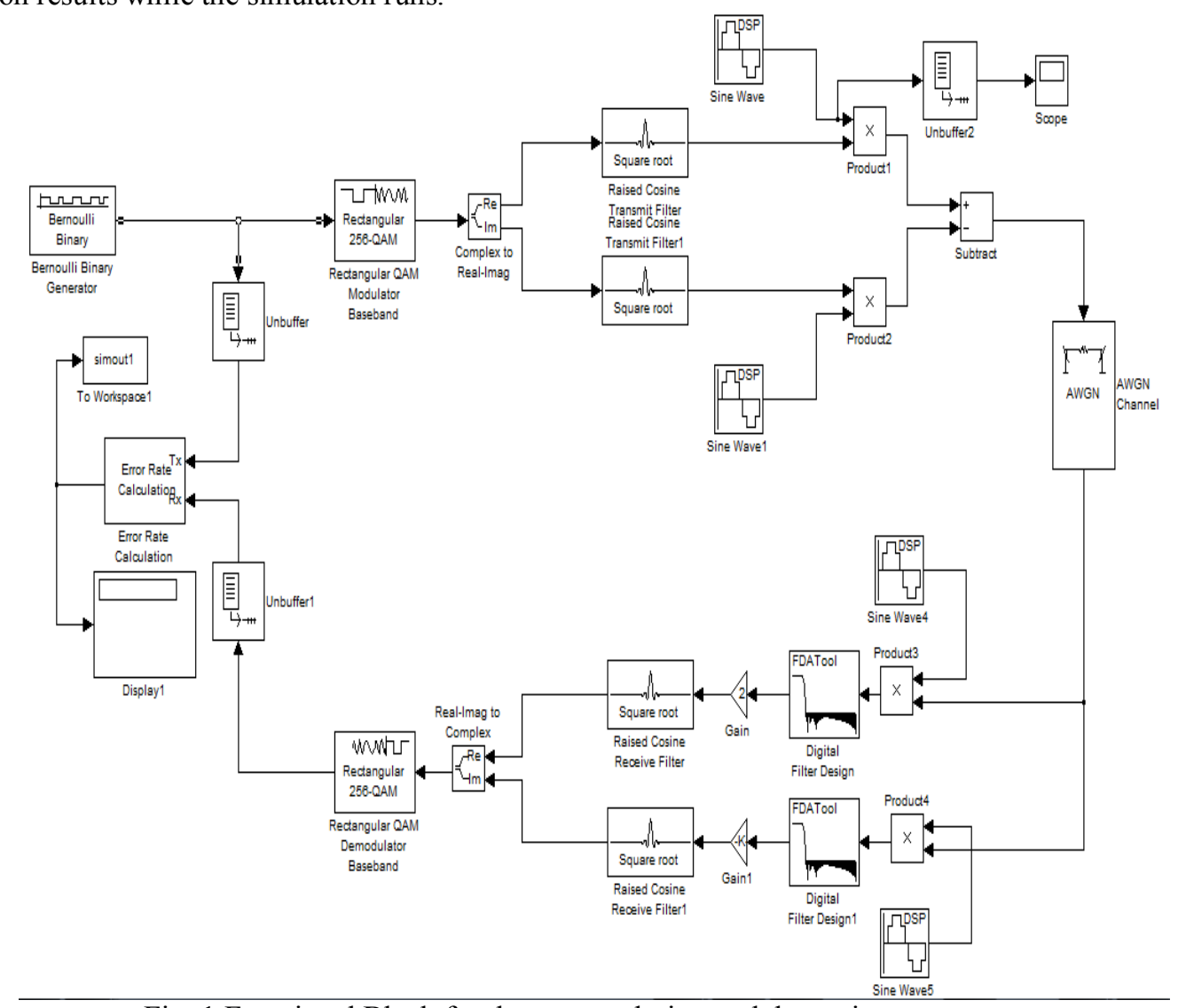

Fig. 1 Functional Block for the system design and the various parameters

\subsection{Spectrum Analysis for 16QAM}

Spectrum analysis was performed on the entire signal for 16QAM as shown below. The modulated signal spectrum is shown in Figure 2. The raised cosine transmit filter block upsampled and filtered the input signal in order to minimise intersymbol interference (ISI) as shown in Figure 3. After passing the data through AWGN channel, it was observed that the transmitted signals were corrupted with noise. The noise in the transmission medium disturbed the signal and caused data corruption as shown in Figure 4. The corrupted signal was passed through a low-pass filter to remove unwanted signal components to enhance the desired signal components as shown in Figure 5. The received cosine filter block filtered the input signal using normal raised cosine FIR filter as shown in Figure 6. Finally, the received signal strength is based on a number of factors including the output of the transmitter, sensitivity of the receiver, path loss and attenuation of the signal as it 
travels through the air from the transmitter to the receiver as shown in Figure 7. The spectrum analysis for the other QAM orders (32QAM, 64QAM, 128QAM, AND 256QAM), also had similar pattern.

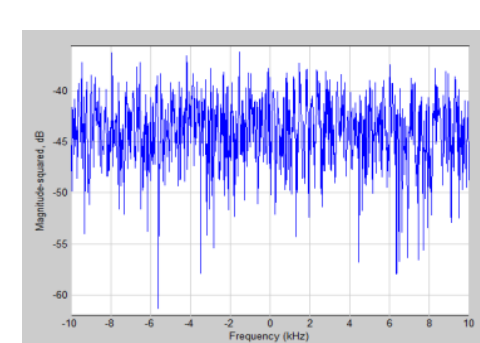

Fig.2: Modulated Signal Sectrum

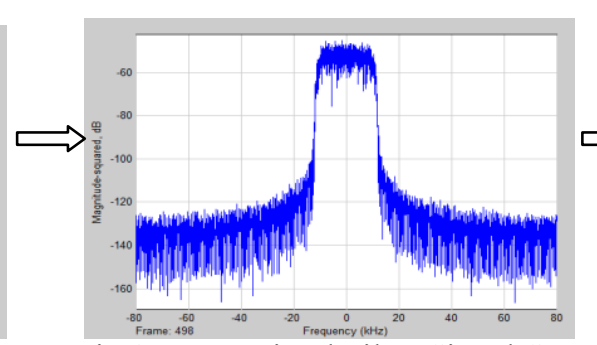

Fig.3: Transmitted Filter Signal Spectrum

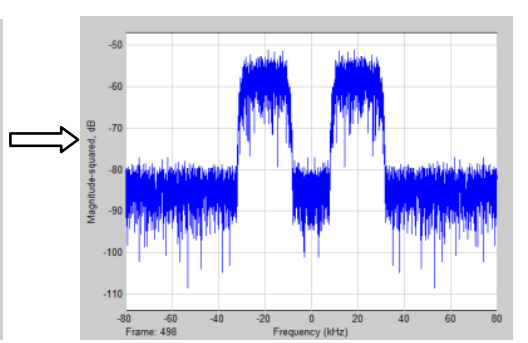

Fig.4: AWGN Channel

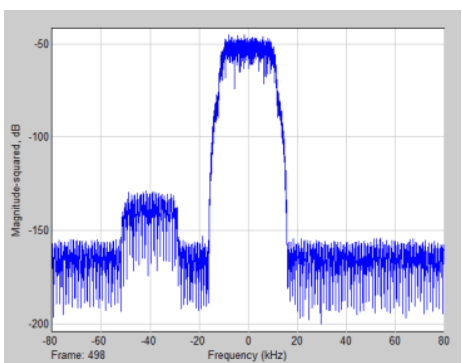

Fig.5 After Low Pass Filter

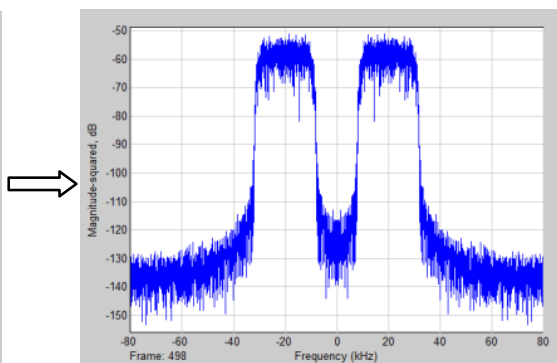

Fig.6 Up-conversion Spectrum

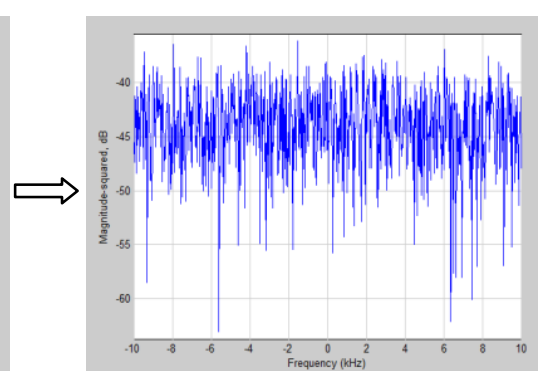

Fig.7 Received Filtered Signal

Signal Spectrum

Performance analysis was done for different QAM orders by passing through Gaussian channel which adds additive white Gaussian noise (AWGN) to the channel. Through the use of simulink, the QAM orders were run simultaneously when $E_{b} / N_{0}$ was $12.0 \mathrm{~dB}$ and the simulated results are shown below.

\section{Ber For Qam Orders When $E_{b} / N_{0}$ IS 12.0db}

Table 1: BER for QAM Orders when $E_{b} / N_{0}$ is $12.0 \mathrm{~dB}$

\begin{tabular}{|c|c|}
\hline Modulation Orders & Simulated BER \\
\hline 16QAM & 0.0001387 \\
\hline 32QAM & 0.0044040 \\
\hline 64QAM & 0.0097240 \\
\hline 128QAM & 0.0383100 \\
\hline 256QAM & 0.0520800 \\
\hline
\end{tabular}

\section{Comparison Of Simulated Result And Theoretical Result}

Table 2: QAM Orders for theoretical and simulated BER

\begin{tabular}{|c|c|c|}
\hline Modulation Orders & Theoretical BER & Simulation BER \\
\hline 16QAM & 0.0001403 & 0.0001387 \\
\hline 32QAM & 0.0018190 & 0.0044040 \\
\hline 64QAM & 0.0096780 & 0.0097240 \\
\hline 128QAM & 0.0276300 & 0.0383100 \\
\hline 256QAM & 0.0521000 & 0.0520800 \\
\hline
\end{tabular}

In the simulation platform, the different QAM orders were used to obtain BER performance curves in AWGN channel. When QAM orders for $E_{b} / N_{0}$ were set at $12.0 \mathrm{~dB}$, the new simulated BER's for the different QAM's were obtained. It was seen from Table 1 shown above that as the QAM order increased; the simulated BER values also increased indicating that the modulated order is directly proportional to the simulated BER. The higher the QAM order, the higher the simulated BER value. The simulated BER's obtained from the generated code were used to generate a graph and compared to the theoretical graph as shown in Figure 8 below. Generally, it was observed that the QAM orders with even bits; (16QAM, 64QAM and 256QAM) had a very small BER whiles the QAM orders with odd bits; (32QAM and 128QAM) had a larger BER. This was clear in the simulated and theoretical graphs shown below. The higher the $E_{b} / N_{0}$ value, the better the BER based on data generated by the computer simulation model. This is because the symbol points get closer as the order of QAM increased if the transmission power is constant. 


\subsection{Comparism of the Communication Systems Performance (Graph)}

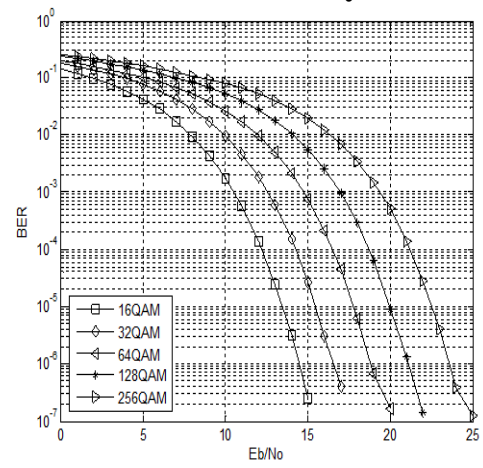

Fig.8: Simulated Graph

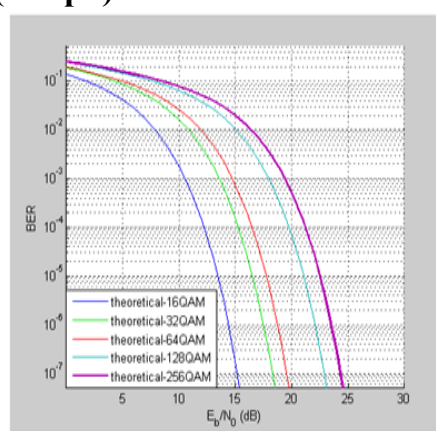

Fig.9: Theoretical Graph

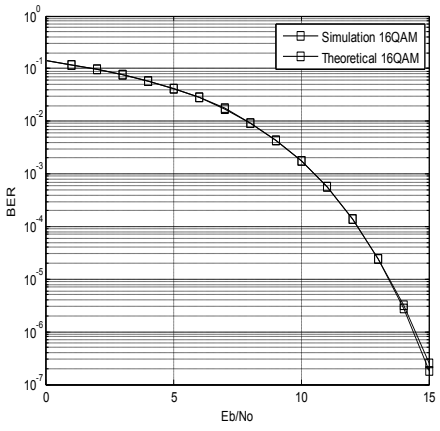

Fig.10 Comparison of Simulated and Theoretical Plot for 16QAM

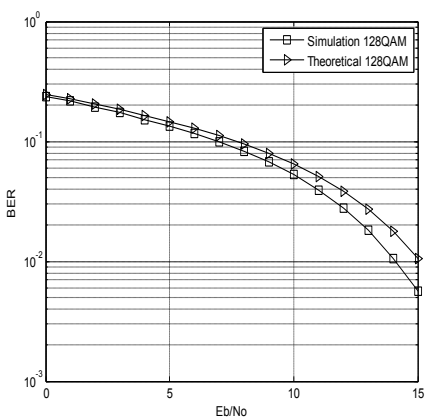

Fig.14 Comparism of Simulated and Theoretical Plot for 128QAM

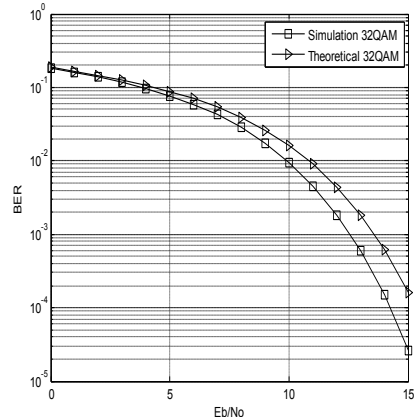

Fig. 11 Comparison of Simulated and Theoretical Plot for 32QAM

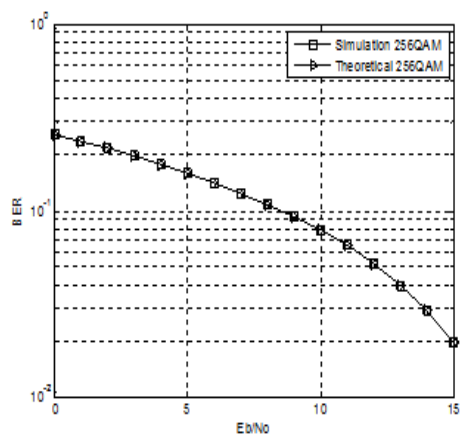

Fig.14 Comparism of Simulated and Theoretical Plot for 256 QAM

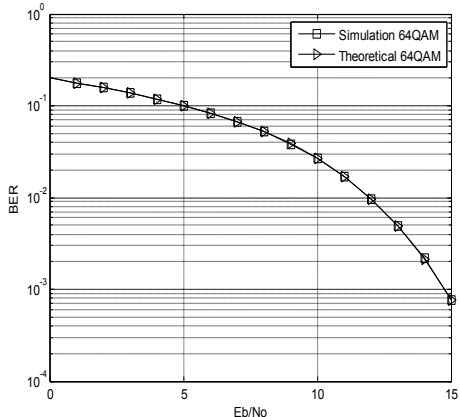

Fig. 12 Comparison of Simulated and Theoretical Plot for 64QAM

\section{Conclusion}

This paper presented a system design that incorporated the functionalities of a digital modulator/demodulator along with controlling features such as symbol generation, filtering, modulation, demodulation, phase and timing recovery for QAM modulation schemes using BER of $10^{-4}$ and $E_{b} / N_{0}$ of 12.0dB. The simulated results for the various QAM orders; 16QAM, 32QAM, 64QAM, 128QAM and 256QAM when $E_{b} / N_{0}$ was set at $12 \mathrm{~dB}$ using the generated code were 0.0001387, 0.004404, 0.009240, 0.038310 and 0.052080 respectively. Comparing the simulated results with the theoretical results, it was found to be almost similar. They both had their BER's increasing as the QAM orders increased with very little significant increase in value of the BER. Nevertheless a wide variety of possible issues occurs primarily due to the nature of the system infrastructure. A comprehensive list of impairments such as thermal noise, reflection paths, frequency response flatness, Interference, group delay variation and phase noise. The simulation model verified that the theory of QAM can be used not only for the criteria for adaptive modulation but also for a platform to design other modulation systems. 


\section{References}

[1] John Proakis, Massoud Salehi, "Digital Communications", McGraw-Hill Education, Nov. 6, 2007.

[2] R. Lucky, J. Salz, and E. Weldon, Principles of Data Communication, New York: McGraw-Hill, 1968.

[3] J. Proakis,(1995). Digital Communications (3rd ed.). McGraw-Hill Inc. ISBN 0-07-113814-5.

[4] Roger Russell, "Hearing, Columns and Comb Filtering". Retrieved 2010-04-22.

[5] Smith, G. Joel, "Odd-Bit Quadrature Amplitude-Shift Keying," IEEE Transactions on Communications, Vol. 23, PP. 385-389, March 1975.

[6] A Leclert and P. Vandamme, "Universal carrier recovery loop for QASK and PSK signal sets", IEEE Transactions on communication, vol. COM-31, PP. 130-36, January 1983.

[7] http://www.trangosys.com/products/point-to-point-wireless-backhaul/licensed wireless/trangolink-apex-orion.shtml 